\section{Effect of Trichloracetic Acid on Adrenaline Chromatograms}

ExTRaCTs free from blood and protein are readily prepared for biological estimation by grinding suprarenal glands with 5 or 10 per cent aqueous trichloracetic acid and centrifuging. However, on submitting such an extract from a cat to paperpartition chromatography using as solvent butanol : acetic acid: water $(4: 1: 5)$, we observed that development with aqueous potassium iodate ${ }^{1}$ gave three spots. Two of these corresponded to adrenaline and noradrenaline $\left(R_{F}=0.32\right.$ and $0 \cdot 25$, respectively) and the third to an unknown adrenaline-like substance $\left(R_{F}=0 \cdot 63\right)$. Three spots were also detected when development was carried out with the Pauly diazoreagent or potassium ferricyanide $(p H \mathrm{H} \cdot 8)$.

Further investigation showed that, when $0.03 \mathrm{ml}$. $(30 \mu \mathrm{gm}$.$) of pure l$-adrenaline in 5 per cent aqueous trichloracetic acid was chromatographed as above, two spots were observed having $R_{F}$ values of 0.32 and 0.63 , respectively. Removal of excess trichloracetic acid from the solution by ether extraction prior to chromatography did not prevent the appearance of the additional spot.

That the effect was due entirely to the presence of trichloracetic acid was shown by passing the solution through a column of the anion exchange resin, 'Amberlite IRA-400', in the chloride form. The filtrate, which now contained adrenaline in approximately $N / 3$ hydrochloric acid, gave only the single adrenaline spot when chromatographed. This spot was equal in intensity to that obtained from a control solution of adrenaline (at the same concentration) in $N / 3$ hydrochloric acid, and biological tests on the cat's blood pressure confirmed that passage of the trichloracetic acid solution through the resin resulted in little or no loss of activity. The accompanying photograph (Fig. 1) shows the chromatogram given by these solutions. When the areas corresponding to the two spots in chromatogram $B$ were eluted with $0.01 \mathrm{~N}$ hydrochloric acid, biological tests showed that the total activity was almost equally divided between the two spots.

The formation of two spots was similarly observed with trichloracetic acid solutions of the following adrenaline-like compounds : 3-hydroxytyramine, epinine, corbasil, $\alpha$-ethylnoradrenaline, $\mathrm{N}$-ethylnor-

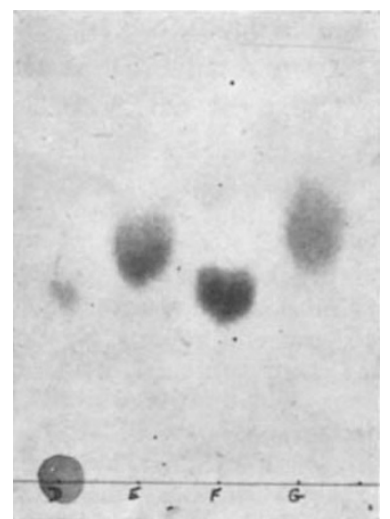

Fig. 1. (A) Solution of zdrenaline ( $30 \mu \mathrm{gma}$.) in 5 per cent tri chloracetic acid after i tssage through "Amberlite 1RA-400" (chloride form). (B) Solut in of adrenaline $(30 \mu \mathrm{gm}$.) in $5 \mathrm{per}$ cen trichloracetic acid before Jassage through 'Amberlite 1 RA- 400 ' (chloride form ' $(C)$ Sol tion of adrenaline $(30 \mu \mathrm{gm}$.) in $N / 3$ hy rochloric as $\mathrm{d}$ for comparison with $(\boldsymbol{A})$

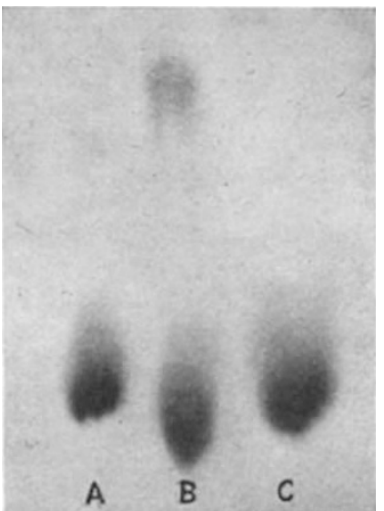

Fig. 2. (D) Adrenaline (30 $\mu \mathrm{gm}$.) in $N / 3$ hydrochloric acid dried at $100^{\circ} \mathrm{C}$. before chromatography. $(E)$ Adrenaline $(30 \mu \mathrm{gm}$.) in $0.01 N$ hydrochloric acid dried at $100^{\circ} \mathrm{C}$. before chromatography.

$(F)$ As $D$, dried at $15^{\circ} \mathrm{C}$. $(G) \mathrm{As} E$, dried at $15^{\circ} \mathrm{C}$.

adrenaline, $\mathrm{N}$-isopropylnoradrenaline and adrenalone. On the other hand, noradrenaline and 3.4-dihydroxyphenylalanine each produced only one spot under the same conditions.

Recently, Crawford ${ }^{2}$ showed that adrenaline and noradrenaline may, under certain conditions, react with lactic acid yielding lactyl derivatives by esterification of their side-chain hydroxyl group; the resulting solutions produced two spots when chromatographed in phenol-water or butanol-hydrochloric acid. However, the fact that in our experiments two spots were obtained with adrenalone, 3-hydroxytyramine and epinine (compounds having no hydroxyl group in the side-chain) indicated that the phenomenon did not arise by formation of a trichloracetic ester.

A possible explanation is furnished by the work of Kendall ${ }^{3}$, who showed that weakly acid phenols can form rather unstable compounds with trichloracetic acid $\left(K=2 \times 10^{-1}\right)$ and with picric acid $\left(K=1 \cdot 6 \times 10^{-1}\right)$. Thus, the additional spot observed in our chromatograms may be due to such a compound of adrenaline with trichloracetic acid. That solutions of adrenaline in 5 per cent methanolic picric acid were also found to give two spots when chromatographed lends support to this view. Two spots have not been observed on chromatographing solutions of adrenaline in 5 per cent aqueous oxalic acid $(K=$ $\left.6 \cdot 5 \times 10^{-2}\right)$, but this acid was not studied by Kendall.

In the course of the above experiments two further phenomena were observed: (1) retardation of the spots when the adrenaline solution was prepared in $N / 3$ instead of $0.01 N$ hydrochloric acid, and (2) retention of material at the starting-line when the applied spots were dried at elevated temperature prior to chromatography. These points are illustrated in Fig. 2:

The above results emphasize the need for great care in the preparation and interpretation of chromatograms of adrenaline-like compounds in tissue extracts.

D. M. SHEPHERD

G. B. West

Department of Pharmacology and Therapeutics, University of St. Andrews Medical School, Dundee.

Dec. 17.

${ }^{1}$ Shepherd, D. M., and West, G. B., Brit. J. Pharmacol., 6, 665.(1951). ${ }^{2}$ Crawford, T. B. B., Biochem. J., 48, 203 (1951).

s Kendall, J., J. Amer. Chem. Soc., 38, 1309 (1916). 